\title{
Resolving Hydrogen at Metal-metal Hydride Interfaces Using iDPC-STEM
}

Sytze de Graaf ${ }^{1}$, Jamo Momand ${ }^{1}$, Christoph Mitterbauer ${ }^{2}$, Sorin Lazar $^{2}$ and Bart Kooi ${ }^{1}$

${ }^{1}$ Zernike Institute for Advanced Materials, Groningen, Groningen, Netherlands, ${ }^{2}$ Thermo Fisher Scientific, Eindhoven, Noord-Brabant, Netherlands

Hydrogen plays an important role in materials science. For example, hydrogen can be stored safely with high volumetric density in metals, however, its absorption in metals can also have disastrous consequences due to hydrogen embrittlement. Yet, imaging hydrogen with scanning transmission electron microscopy has remained elusive, despite the very high spatial resolution that can be achieved nowadays. Here we show that hydrogen can be imaged with integrated differential phase contrast (iDPC) in an aberration corrected scanning transmission electron microscope (1).

We study the $\gamma$-titanium monohydride precipitates, which are often present even in pure titanium, because they readily form due to the very low hydrogen solubility limit in titanium at room temperature. The $\gamma$ titanium monohydride has a tetragonal unit cell in which hydrogen occupies half of the tetrahedral sites, and the other half is in principle empty. A planar coherent interface is formed between the hexagonal close-packed titanium and the tetragonal $\gamma$-titanium monohydride despite the large unit cell volume difference of about $15 \%$. This volume misfit is entirely accommodated by a $15 \%$ lattice mismatch perpendicular to the interface, resulting in large internal stresses in the material (2). Three models that describe the possible position of the hydrogen atoms at the interface have been proposed in the 1980s, as at that time only the titanium lattice could be imaged using high-resolution transmission electron microscopy (3).

In this work we have imaged the interface between the titanium and $\gamma$-titanium monohydride using highangle annular dark-field (HAADF), annular bright-field (ABF) and iDPC STEM. It was only possible to image the interface in parts of the specimen thicker than about $30 \mathrm{~nm}$. The $\gamma$-titanium monohydride was found to be instable in the thinnest parts, leading to amorphization during sample preparation. Slightly thicker parts were subjected to substantial bending and buckling on a nanometer scale that prevented us from aligning the crystal.

We have unambiguously imaged the hydrogen atoms at the titanium/ $\gamma$-titanium monohydride interface with the iDPC-STEM technique (Figure 1). Particularly the lower signal-to-noise ratio (SNR) and the sensitivity towards crystal misorientation prevented reliable imaging of hydrogen with ABF-STEM. Exploiting the fact that this monohydride has empty columns and hydrogen occupied columns with identical surrounding of the host titanium atom we have, 30 years after they were first proposed, finally resolved which one of the proposed structural models holds for the interface.

High quality images of the $\gamma$-titanium monohydride have been acquired in thicker parts of the specimen and further away from the interface, where bending and buckling are minimized (Figure 2). There the superior image quality of iDPC compared with $\mathrm{ABF}$ is emphasized, as the image constructed from the exact same detector data show that the SNR is 2-3 times higher in the iDPC image. Our work enables new, previously impossible, research on hydrides and is extendable to all materials containing light and heavy elements, including oxides, nitrides, carbides, and borides. 

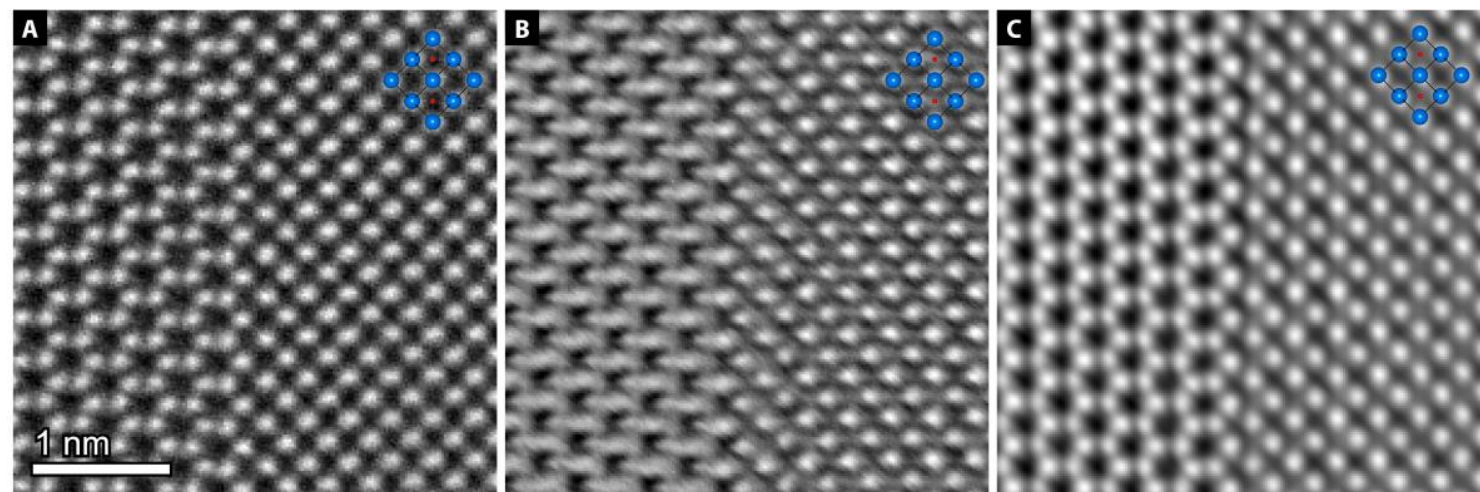

Figure 1. Images of the titanium $/ \gamma$-titanium monohydride interface using (A) HAADF, (B) ABF, (C) iDPC STEM, where only iDPC allows unambiguous detection of hydrogen atomic columns.
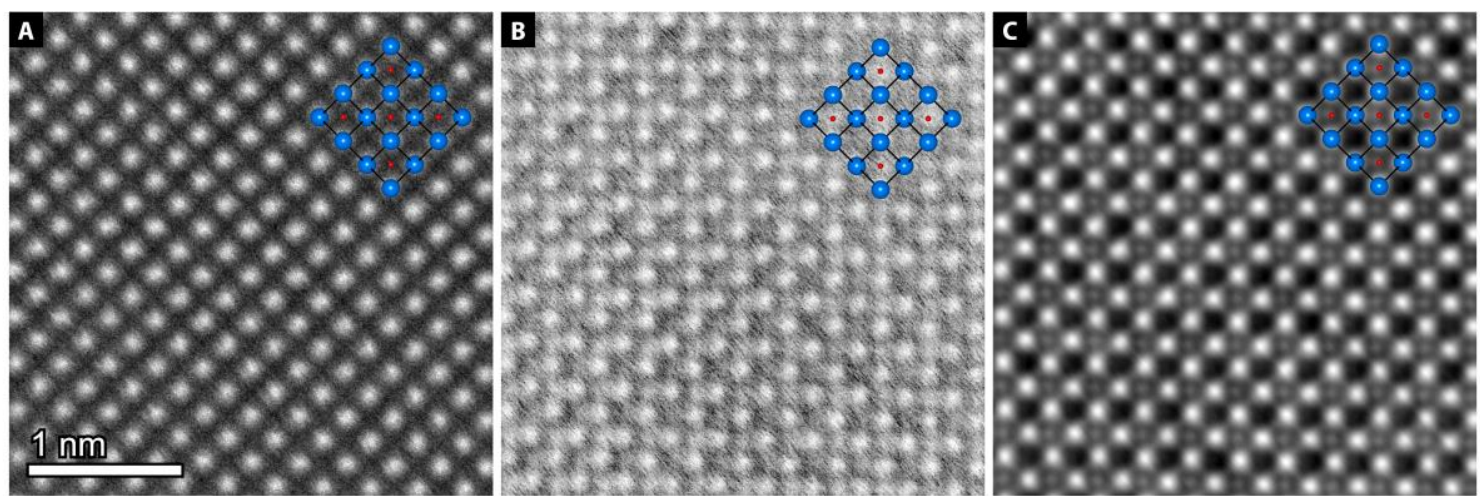

Figure 2. Images of the $\gamma$-titanium monohydride using (A) HAADF, (B) ABF, (C) iDPC STEM, where the superior capability of iDPC to detect hydrogen atomic columns is demonstrated.

\section{References}

1. S. de Graaf, J. Momand, C. Mitterbauer, S. Lazar, B.J. Kooi. Science Advances. 6 (2020).

2. H. Numakura, M. Koiwa, Hydride precipitation in titanium. Acta Metallurgica. 32, 1799-1807 (1984). 3. A. Bourret, A. Lasalmonie, S. Naka, In-situ high resolution observation of hydride precipitation in titanium. Scripta Metallurgica. 20, 861-866 (1986). 